\title{
POSSIBILITIES OF SIMULATION MODELS VISUALIZATION IN TEACHING PROCESS
}

\author{
Peter Grabusts \\ Rezekne Academy of Technologies, Latvia
}

\begin{abstract}
Teaching experience shows that during educational process student perceive graphical information better than analytical relationships. Many educational courses operate with models that were previously available only in mathematics and physics disciplines. As a possible solution, there could be the use of package Matlab Simulink in realization of different algorithms both for engineering disciplines and economic studies. The paper presents examples of using simulation modelling in the educational research processes.
\end{abstract}

Keywords: Matlab Simulink, modelling, simulation, teaching, visualization.

\section{Introduction}

The aim of the study of different simulation models is the formation students' theoretical knowledge and practical skills in the use of simulation modelling techniques in research of solving specific problems in programming and modelling of real applications - matrix algebra, solving of equations, economics applications. During the course students get acquainted with the means of simulation modelling processes of functioning systems, master the simulation modelling methods and typical stages of modelling process that form the ,chain": the construction of the conceptual model and its formalization model algorithmization and its computer implementation - simulation experiment and interpretation of the results of the modelling; acquire practical skills for implementation of modelling algorithms for studies of characteristics and behaviour of complex economic systems (Kay, 1984; Karel \& Tomas, 2015).

As a result, students acquire knowledge about:

- Basics of theory and practice of simulation modelling;

- The main classes of models of domain systems, their modelling technology.

They should be able to:

- Use the simulation modelling method in studying and designing of different systems; 
- Develope schemes of modelling algorithms of processes and systems, implement the models using the simulation software package Matlab Simulink.

Simulation - the most powerful and versatile method for studying and evaluating the effectiveness of systems, the behaviour of which depends on the influence of random factors.

The implementation of such opportunities in the universal programming language is a difficult task. Currently, there are quite a lot of software, that allow to model processes. However, now there is a product that allows to solve these problems quite effectively - MATLAB packet (Kiusalaas, 2016; Siauw \& Bayern, 2015; Smith, 2013) containing visual simulation tool - Simulink (http://se.mathworks.com/products/simulink/). Simulink - a tool that allows you to simulate the system quickly, get the indexes of expected effect and compare them with the amount of effort required to achieve them (Karel \& Tomas, 2015; Xue \& Chen, 2013).

\section{Possibilities of the software package Matlab Simulink}

Of particular interest for simulation is a Simulink tool designed specifically for modeling dynamical systems. It has a library of standard graphics units with built-in mathematical functions. It is sometimes called a tool of visual modeling (Shiflet \& Shiflet, 2014; Silva, 2009).

Although Simulink is designed mainly to solve engineering and scientifictechnical problems, the possibilities of its use are almost unlimited. The input of initial parameters is made interactively by graphics assembly of elementary blocks circuit diagram, resulting in a model of the studied system. The blocks included in the model relate to each other both in information and in management. The type of connection depends on the type of the block and the logic of the model.

Simulink program is an application to the MATLAB package. In the simulation using Simulink is implemented the principle of visual programming whereby the user on the screen creates a model of a structure, process or system from standard blocks of the library, and performs calculations. In this case, unlike in classical ways of modeling, the user does not need to study the programming language and numerical mathematics methods thoroughly, there is enough to have some general knowledge required when working on the computer, and, of course, knowledge on the subject area in which he works.

Creating a model in this way, then launching it, it is possible to see the results of modeling.

In the simulation the user can choose the method for solving equations, as well as the way to change the model time (with fixed or variable step). During 
the simulation it is possible to monitor the processes happening in the system. To this purpose special viewing devices that are part of the Simulink library are used. The simulation results can be presented in the form of graphs or tables.

Series of research were carried out in order to demonstrate the Simulink suitability for simulation model visualization purposes in different engineering disciplines. It should be noted that often the analytical solution is much simpler than the visual Simulink model, but in perspective it gives an understanding of such models usefulness.

Fig. 1 shows the used blocks description and its explanation given in the examples.
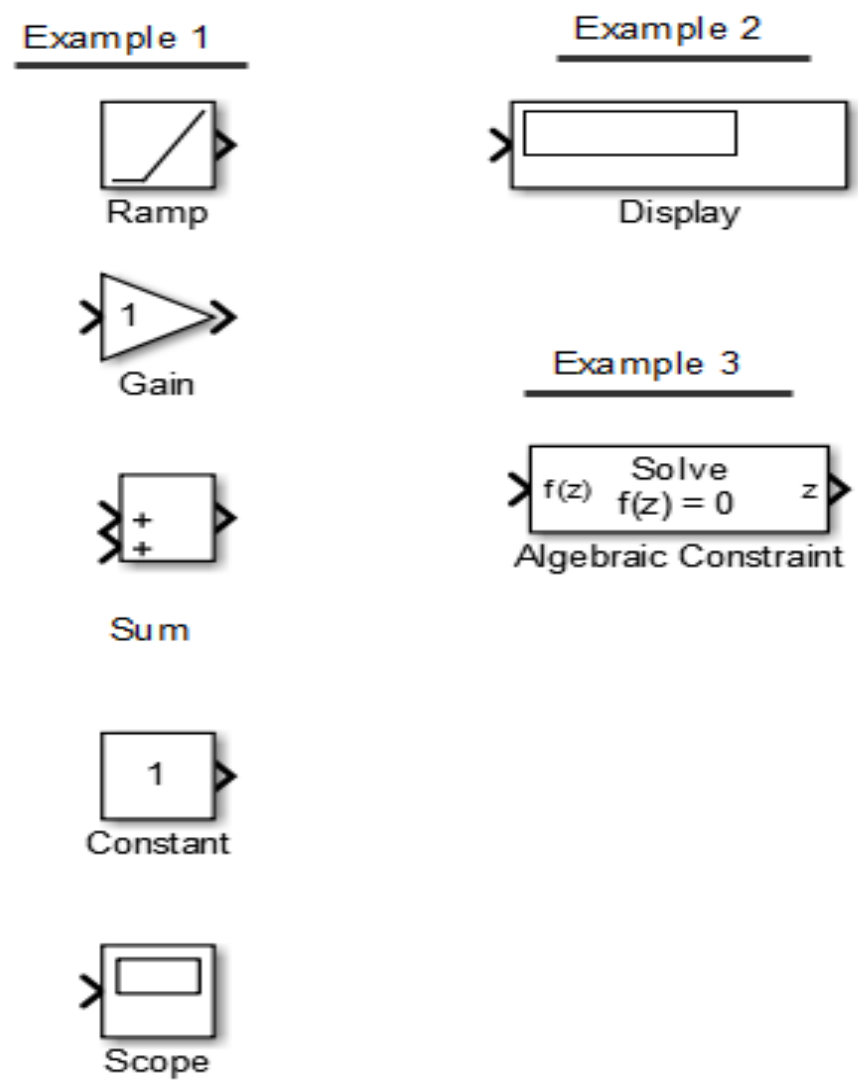

Figure1 Blocks used in the examples

The Ramp block (Sources) generates a signal that starts at a specified time and value and changes by a specified rate.

The Gain block (Common Used Blocks) multiplies the input by a constant value (gain). The input and the gain can each be a scalar, vector, or matrix.

The Sum block (Common Used Blocks) performs addition or subtraction on its inputs. This block can add or subtract scalar, vector, or matrix inputs.

The Constant block (Sources) is used to define a real or complex constant value. 
The Scope block (Common Used Blocks) displays waveforms as functions of simulation time. The Scope block can have multiple $\mathrm{y}-$ axes with a common time range.

The Display block (Sinks) shows the output value at the end of the simulation time.

The Algebraic Constraint block (Math operations) constrains the input signal to zero and outputs an algebraic $f(z)$ state. The block outputs the value necessary to produce a zero at the input. The output must $\mathrm{z}$ affect the input through a feedback path.

\section{Research part}

Example 1. Converting Celsius to Fahrenheit

To model the equation that converts Celsius temperature to Fahrenheit:

$$
T_{F}=9 / 5\left(T_{C}\right)+32 .
$$

The conversion formula can be easily realized by the calculator, or simple application in any programming language. But it could be interesting to see its realization with the help of Simulink (Esfandiari, 2013).

First, consider the blocks needed to build the model:

- $\quad$ Ramp block to input the temperature signal;

- Constant block to define a constant of 32;

- $\quad$ Gain block to multiply the input signal by $9 / 5$;

- Sum block to add the two quantities;

- Scope block to display the output results.

Next, gather the blocks into model window (see Fig. 2).

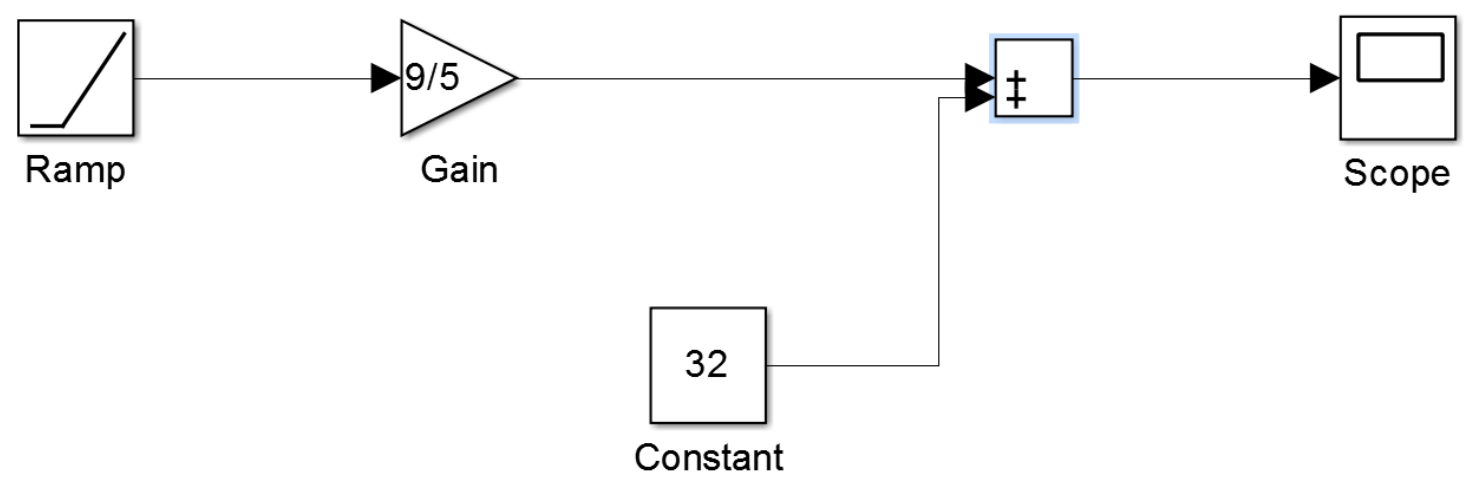

Figure 2 Converting Celsius to Fahrenheit

The Ramp block inputs Celsius temperature. Open that block and change the Initial output parameter to 0 . The Gain block multiplies that temperature by the constant 9/5. The Sum block adds the value 32 to the result and outputs the 
Fahrenheit temperature. Simulation results see in Fig. 3.

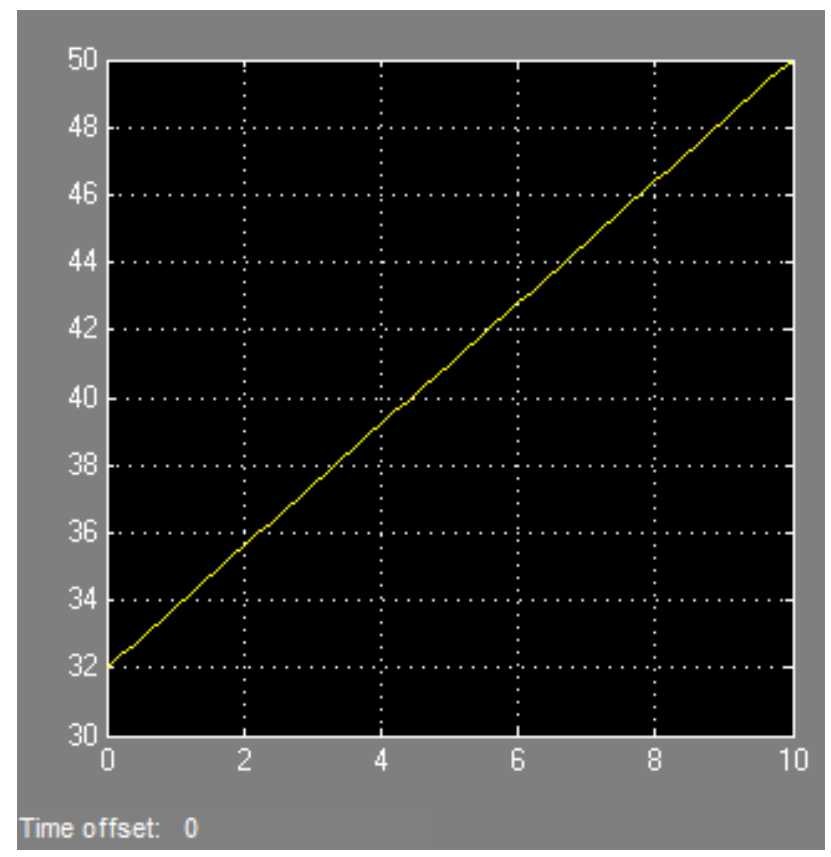

Figure 3 Model simulation results

It can be concluded that between Celsius and Fahrenheit degrees there is a linear relationship.

Example 2. Matrix addition

Matrix addition are taught at higher mathematics course in many engineering specialties. Also in Matlab setting there is a simple formula to obtain the result, but Simulink tools enable to display this operation with the graphic visualization tools, instantly displaying the result after simulation (Karris, 2006).

The matrices $A$ and $B$ are defined as shown. We will create a model using the Sum block to $A+B=C$.

$$
A\left|\begin{array}{lll}
1 & 2 & 3 \\
4 & 5 & 6 \\
7 & 8 & 9
\end{array}\right|+B\left|\begin{array}{lll}
9 & 8 & 7 \\
6 & 5 & 4 \\
3 & 2 & 1
\end{array}\right|=C\left|\begin{array}{lll}
10 & 10 & 10 \\
10 & 10 & 10 \\
10 & 10 & 10
\end{array}\right|
$$

The model is shown in Fig. 4 where in the Matlabs's command window we

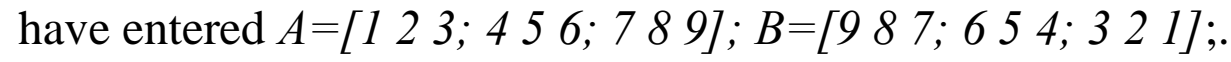




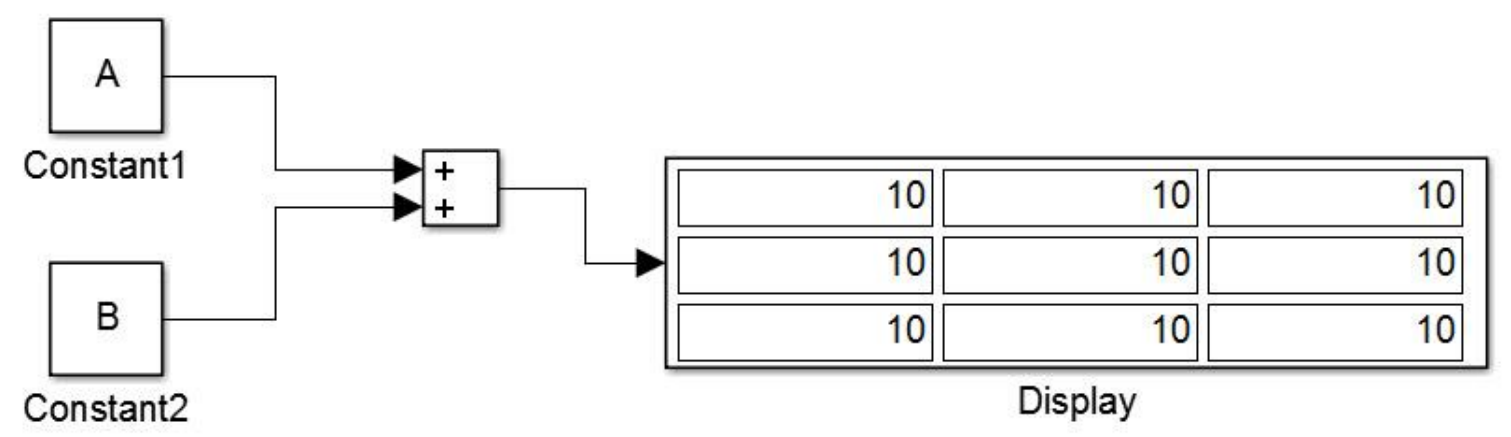

Figure 4 Result of matrix addition model operating

Example 3. Solving equations

Equations are already solved at secondary school. Matlab Simulink enables to show the links between the elements of the equation in graphical form and show the obtained results in demonstrative way (Karris, 2006).

Using Algebraic Constraint block, Display block and Gain block, we will create a model that will produce the simultaneous solution of three equations with three unknowns.

The model will display the values for the unknowns $z_{1}, z_{2}$ and $z_{3}$ in the system of the equations:

$$
\begin{aligned}
& a_{1} z_{1}+a_{2} z_{2}+a_{3} z_{3}+k_{1}=0 \\
& a_{4} z_{1}+a_{5} z_{2}+a_{6} z_{3}+k_{2}=0 \\
& a_{7} z_{1}+a_{8} z_{2}+a_{9} z_{3}+k_{3}=0
\end{aligned}
$$

The model is shown in Fig. 5.

Next, we go to Matlabs's Command window and we enter the following values: $a 1=4 ; a 2=-6 ; a 3=-2 ; a 4=2 ; a 5=10 ; a 6=8 ; a 7=-12 ; a 8=2 ; a 9=4$; $k 1=-16 ; k 2=-14 ; k 3=10$;. After clicking on the simulation icon, we observe the values of the unknowns as $z_{1}=2, z_{2}=-3$ and $z_{3}=5$. These values are shown in the Display block of Fig. 5. 


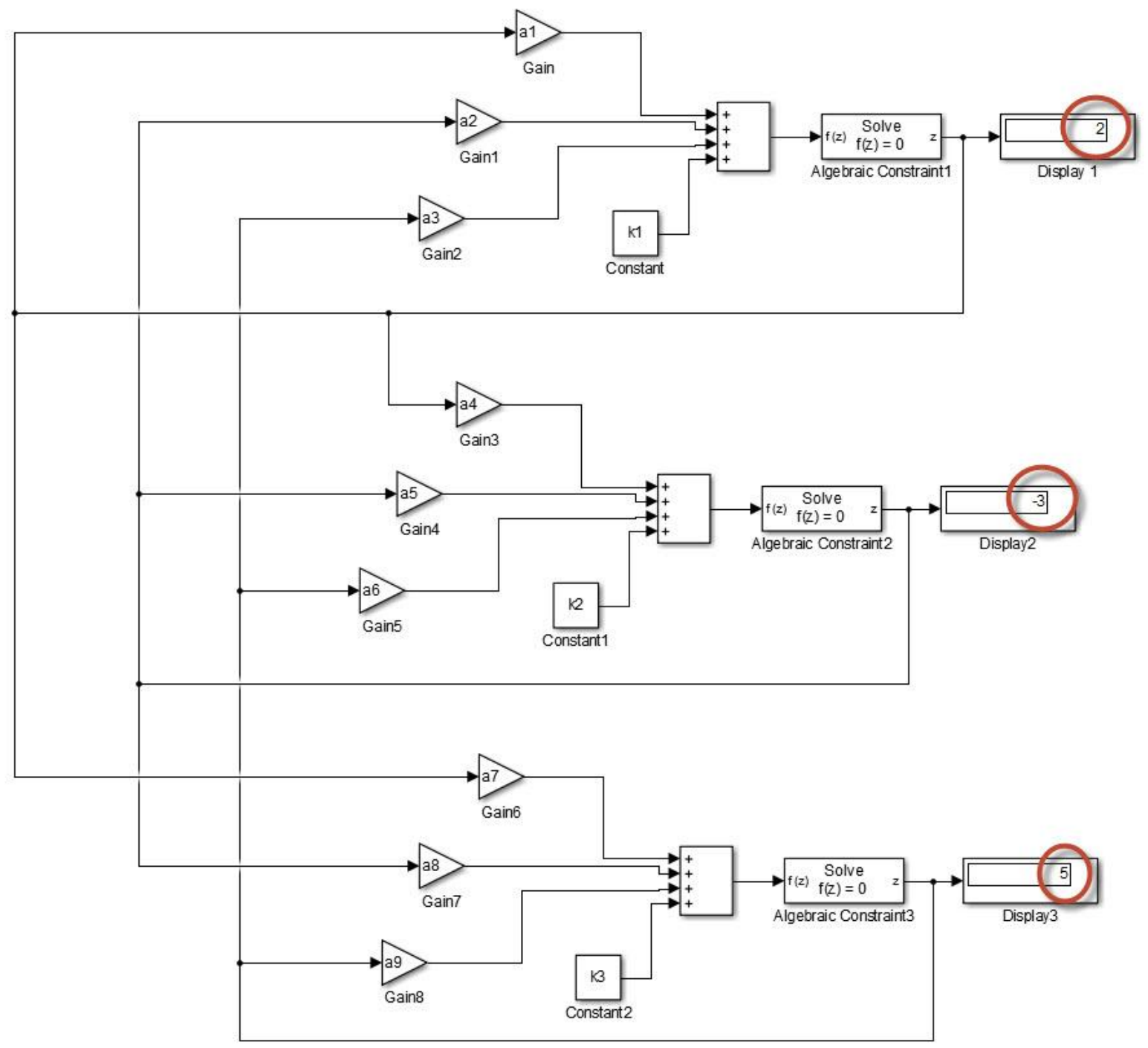

Figure 5 Model of three equations with three unknowns

Although in this case, the visual model may seem complicated, but its further understanding makes it possible to create complex system models - not only in engineering, but also in economics, environmental sciences, sociological studies, and so on (Anderson, 2005; Cerný, 2009; Chapra \& Canale, 2015).

\section{Conclusions}

In this paper the author substantiates the usefulness of introduction of simulation model for the initial teaching process, when parallelly with acquisition of analytic relationship there may also be imported mathematical simulation models. It gives students the opportunity to receive not only the options of using formulas, but also visualize different correlations in graphical form. 
In the research part with the help of some examples the visualization of a simple mathematical formula calculation is demonstrated, the example of matrix addition is given, as well as the equation systems calculation. Of course, these examples can be realized quite simply using specific environmental functions, but the author is assured that the use of simulation models makes it possible to raise learners' horizon and gives an idea of the potential uses of such models.

It can be concluded that Matlab Simulink tool is a very suitable tool not only in engineering calculations, but also can serve as a simulation model visualization tool in various science fields.

\section{References}

Anderson, P.L. (2005). Business Economics and Finance with MATLAB®, GIS, and Simulation Models. Chapman \& Hall.

Cerný, A. (2009). Mathematical Techniques in Finance: Tools for Incomplete Markets, $2 e$. Princeton University Press.

Chapra, S.C., Canale, R.P. (2015). Numerical Methods for Engineers, 7e. McGraw-Hill.

Esfandiari, R.S. (2013). Numerical Methods for Engineers and Scientists Using MATLAB. Chapman \& Hall/CRC.

Kay, C. (1984). Mathematics for Computer Programmers. New Jersey: Prentice Hall.

Karel, P., Tomas, Z. (2015). Multimedia Teaching Aid for Students of Basics of Control Theory in Matlab and Simulink. Procedia Engineering, Volume 100, 150-158.

Karris, S.T. (2006). Introduction to Simulink ${ }^{\circledR}$ with Engineering Applications. Orchard Publications.

Kiusalaas, J. (2016). Numerical Methods in Engineering with MATLAB, 3e. Cambridge University Press.

Shiflet, A.B., Shiflet, G.W. (2014). Introduction to Computational Science: Modeling and Simulation for the Sciences, $2 e$. Princeton University Press.

Siauw, T., Bayern, A. (2015). An Introduction to MATLAB Programming and Numerical Methods for Engineers. Academic Press.

Silva de, C.W., (2009). Modeling and Control of Engineering Systems. CRC Press, Inc.

Smith, D. (2013). Engineering Computation with MATLAB, 3e. Pearson Education Inc.

Xue, D., Chen, Y. (2013). System Simulation Techniques with MATLAB and Simulink. John Wiley \& Sons, Inc. 